using morphological and existing immunohistochemical criteria. Preliminary findings indicate that paediatric glioneuronal tumours may be subclassified based on detection of certain molecular abnormalities. In combination with histopathological analysis, better molecular characterisation of glioneuronal tumours has the potential to improve diagnostic accuracy and provide data relevant for treatment and prognostication.

Aim: To characterise genomic and genetic alterations in paediatric and adult glioneuronal tumours.

Methods: DNA extracted from 30 FFPE low grade glioneuronal tumours was analysed using combinations of low pass whole genome sequencing (LP-WGS) and multiplex ligation-dependent probe amplification (MLPA) for brain tumour associated genetic and genomic alterations.

Results: LP-WGS of 22 glioneuronal tumours detected no whole or partial chromosomal losses or gains in 9 of the tumours. Chromosome 7 gain was identified in 6 of the tumours and was the most common genomic alteration, with other chromosomal abnormalities $(1 \mathrm{p}-, 5+, 8 \mathrm{q}+, 9-, 10 \mathrm{p}+)$ evident in single tumours. Two tumours, an unclassified high grade glioneuronal tumour and a low grade ganglioglioma exhibited multiple chromosomal abnormalities, $1 \mathrm{p}-/ 3 \mathrm{p}-/ 14-/ 15-/ 16 \mathrm{q}-/ 17 \mathrm{q}+$ and $5+/ 7+/ 8+/ 9+/ 10+/ 12+/ 13+/ 16+/ 20+/ 21+$, respectively. MLPA analysis confirmed regions of $7 p$ gain (EGFR) and $8 p$ gain (FGFR1/TACC) and identified BRAF ${ }^{\mathrm{V} 600 \mathrm{E}}$ mutations in 3 tumours. CDKN2A loss, a marker of poor prognosis, was detected in one tumour.

Conclusions: Findings of this study indicate glioneuronal tumours to be a molecularly heterogeneous group of neoplasms that may be subtyped on the basis of chromosomal and genetic alterations, and using laboratory procedures available in diagnostic pathology practice. Refinement of molecular profiling, and the detection of markers of poor prognosis or targetable mutations may improve tumour classification algorithms and enable better management of this rare tumour entity.

\section{ASSOCIATION BETWEEN IGG4-RELATED DISEASE AND DURAL ARTERIOVENOUS FISTULA FORMATION}

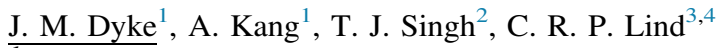

${ }^{1}$ Department of Neuropathology, PathWest, Royal Perth Hospital, Perth, WA, Australia; ${ }^{2}$ Neurological Intervention and Imaging Service of Western Australia (NIISwa), Sir Charles Gairdner Hospital, Nedlands, WA, Australia; ${ }^{3}$ School of Surgery, University of Western Australia, Crawley, WA, Australia; and ${ }^{4}$ Department of Neurosurgery, Sir Charles Gairdner Hospital, Nedlands, WA, Australia

Background: IgG4-related disease (IgG4-RD) includes a variety of diseases characterised by infiltration of the affected organ/ $\mathrm{s}$ by IgG4-positive plasma cells, usually accompanied by fibrosis and obliterative venous damage. Comprehensive diagnostic criteria formulated in 2011 require characteristic organ involvement and one or both of a raised serum IgG4 level and increased numbers of IgG4-positive plasma cells on histopathology. In the central nervous system, one of the most common manifestations of IgG4-RD is pachymeningitis. It has been postulated that calvarial dural arteriovenous fistula (DAVF) formation may be initiated by local inflammation at the penetrating point on the dura of the emissary vein, with vascular remodelling altering the local haemodynamics and resulting in aberrant arteriovenous connections with subsequent fistula formation.

Aims: A report of the co-occurrence of chronic dural inflammation in the form of IgG4-RD and DAVF.

Methods: A single case study and review of the supporting literature.

Results and conclusions: A 54-year-old female presented with recent onset simple partial and generalised secondary seizures. MRI brain and cranial angiogram found a meningeal-diploic vein fistula supplied by the left middle meningeal, anterior deep temporal and middle deep temporal arteries. Cortical venous hypertension was present with associated haemorrhagic oedema of the left frontal lobe. The patient had an open resection of the DAVF after unsuccessful attempted fistula embolisation. Histopathology on dura adjacent to the calvarial fistula showed dural fibrosis, focal obliterative phlebitis and chronic inflammation including numerous plasma cells and lymphocytes. On immunohistochemical staining up to $53 \mathrm{IgG} 4$ positive plasma cells were present per high power field, with an IgG:IgG4 ratio of $42.4 \%$. These findings meet the histopathological criteria for IgG4-related disease (IgG4-RD).

This case supports the association of chronic dural inflammation and DAVF and is the first reported co-occurrence of IgG4-RD and DAVF.

\section{LOCALISED PRIMARY AMYLOIDOSIS OF THE BLADDER MIMICKING CARCINOMA}

\author{
A. Busuttil $^{1}$, A. Moghimi ${ }^{2}$ \\ ${ }^{1}$ Footscray Hospital, Melbourne, Australia; and ${ }^{2}$ Monash \\ Medical Centre, Melbourne, Australia
}

Background: Amyloidosis is a heterogeneous pathologic process, the hallmark of which is the extracellular deposition of a proteinaceous substance, which has characteristic histologic features. Although rare in the lower urinary tract, its presence in the bladder has been reported, and can clinically mimic carcinoma. Aims: We herein present the case of a middle aged man diagnosed with localised primary amyloidosis of the bladder whose clinical presentation raised concern for carcinoma. We discuss the histopathologic manifestations of amyloid deposition in the bladder with important pathologic diagnostic considerations and their clinical sequelae.

Methods: A clinico-pathologic review of a case of localised primary amyloidosis of the bladder in which a 57-year-old man presented with significant haematuria and a solid lesion at cystoscopy, thought to be urothelial carcinoma. Light microscopic features of the lesion, in conjunction with ancillary tests inclusive of special stains, immunohistochemistry, in situ hybridisation and serologic studies are described and discussed. Results and discussion: Microscopy showed polypoid fragments of bladder mucosa, the stroma of which contained extensive deposits of eosinophilic, amorphous hyaline material which appeared pink/red with a Congo red stain and showed the characteristic green birefringence of amyloid under polarised light. An established diagnosis of amyloidosis, as in this case, requires further information to be of clinical tenor. Important considerations include whether the deposition is localised or systemic in nature as well as typing of the amyloid. Addressing the former typically requires clinical correlation and is of import as localised disease does not require systemic therapy and, in contrast to systemic amyloidosis, has excellent long term prognosis. Vesical 